Commun. Korean Math. Soc. 28 (2013), No. 3, pp. 559-569

http://dx.doi.org/10.4134/CKMS.2013.28.3.559

\title{
REGULARITY FOR SEMILINEAR RETARDED FUNCTIONAL INTEGRODIFFERENTIAL EQUATIONS
}

\author{
Jin-Mun JeOng And Su Jin Cheon
}

\begin{abstract}
The purpose of this paper is to establish existence, uniqueness and a variation of constant formula of solutions for semilinear partial functional differential equations with unbounded delays and nonlinear part involving integrodifferetial terms.
\end{abstract}

\section{Introduction}

This paper is concerned with the existence, uniqueness and norm estimations of solutions for a class of partial functional integrodifferential systems with delay terms:

$$
\left\{\begin{aligned}
& \frac{\partial}{\partial t} u(t, x)+\mathcal{A}\left(x, D_{x}\right) u(t, x)+\mathcal{A}_{1}\left(x, D_{x}\right) u(t-h, x) \\
& +\int_{-h}^{0} a(s) \mathcal{A}_{2}\left(x, D_{x}\right) u(t+s, x) d s-f(t, x) \\
= & F\left(t, u(t-h, x), \int_{0}^{t} k(t, s, u(s-h, x)) d s\right),(t, x) \in[0, T] \times \Omega .
\end{aligned}\right.
$$

Here, $\Omega \subset \mathcal{R}^{n}$ is a bounded domain with smooth boundary $\partial \Omega, \mathcal{A}\left(x, D_{x}\right)$ and $\mathcal{A}_{\iota}\left(x, D_{x}\right)(\iota=1,2)$ are second order linear differential operators with real coefficients, and $\mathcal{A}\left(x, D_{x}\right)$ is an elliptic operator in $\bar{\Omega}$. The function $a(s)$ is a real scalar function on $[-h, 0]$, where $h>0$ is a delay time and $f$ is a forcing function. The boundary condition attached to (1.1) is given by Dirichlet boundary condition

$$
\left.u\right|_{\partial \Omega}=0, \quad 0<t \leq T,
$$

and the initial condition is given by

$$
u(0, x)=g^{0}(x), \quad u(s, x)=g^{1}(s, x) \quad-h \leq s \leq 0 .
$$

Received September 26, 2012; Revised February 19, 2013.

2010 Mathematics Subject Classification. Primary 35F25, 35K55.

Key words and phrases. semilinear equation, retarded functional integrodifferential equation, existence of solution, uniqueness of solution, norm estimation.

This research was supported by Basic Science Research Program through the National research Foundation of Korea(NRF) funded by the Ministry of Education, Science and Technology(2012-0007560). 
Set

$$
G(t, u)=F\left(t, u(t-h), \int_{0}^{t} k(t, s, u(s-h)) d s\right)
$$

The nonlinear term $G(t, \cdot)$, which is a Lipschitz continuous operator from $L^{2}(-h, T ; V)$ to $L^{2}(-h, T ; H)$, is a semilinear version of the quasilinear one considered in Yong and Pan [9]. Precise assumptions are given in the next section.

The abstract formulations of many partial integrodifferential equations arise in the mathematical description of the dynamical processes with heat flow in material with memory, viscoelasticity, and many physical phenomena (see $[3,4])$. When $F \equiv 0$ in $(1.1)$, this linear type of equations is studied extensively by Di Blasio et al. [2], Tanabe [7] and Jeong, Nakagiri [5, 6]. Most parts of previous results studied the regularity for nonlinear equations under conditions of the uniform boundedness of the nonlinear terms and the compactness of the principal operators.

The purpose of this paper is to establish a variation of constant formula and regularity property of solutions for the equation (1.1) with the aid of intermediate theory and the regularity for the corresponding linear equation(in case $F \equiv 0$ ). We can also see that the solution mapping $f \mapsto x_{u}$ is compact where $x_{f}$ is a solution of (1.1) corresponding to the forcing term $f$ which is an important rule to apply control and optimal problems.

In order to prove the solvability of the initial value problem (1.1) we establish necessary estimates applying the result of [2] to (1.1) considered as an equation in a Hilbert space. In this paper, we give preliminaries on linear equations, and then prove the local existence and uniqueness for solution of (1.1)-(1.3) by using the contraction principle. Finally, we establish the norm estimation of solutions by using the regularity for solutions associated with the linear part of the given equations and the global existence of solutions by the step by step method.

\section{Preliminaries and local solutions}

Let $H$ and $V$ be two complex Hilbert spaces such that $V$ is a dense subspace of $H$. The norm of $H$ (resp. $V$ ) is denoted by $|\cdot|$ (resp. $\|\cdot\|$ ) and the corresponding scalar product by $(\cdot, \cdot)$ (resp. $((\cdot, \cdot)))$. Assume that the injection of $V$ into $H$ is continuous. The antidual of $V$ is denoted by $V^{*}$, and the norm of $V^{*}$ by $\|\cdot\|^{*}$. Identifying $H$ with its antidual we may consider that $H$ is embedded in $V^{*}$. Hence we have $V \subset H \subset V^{*}$ densely and continuously.

We realize the operator $\mathcal{A}\left(x, D_{x}\right), \mathcal{A}_{\iota}\left(x, D_{x}\right), \iota=1$, 2 , in Hilbert spaces by

$$
A_{0} v=-\mathcal{A}\left(x, D_{x}\right) v, \quad A_{\iota} v=-\mathcal{A}_{\iota}\left(x, D_{x}\right) v, \iota=1,2, \quad v \in V
$$


in the distribution sense. The mixed problem (1.1) can be formulated abstractly as

(SLE)

$$
\left\{\begin{aligned}
\frac{d}{d t} u(t)= & A_{0} u(t)+A_{1} u(t-h)+\int_{-h}^{0} a(s) A_{2} u(t+s) d s \\
& +F\left(t, u(t-h), \int_{0}^{t} k(t, s, u(s-h)) d s\right)+f(t), \quad 0 \leq t \leq T \\
u(0)= & g^{0}, \quad u(s)=g^{1}(s), \quad-h \leq s \leq 0 .
\end{aligned}\right.
$$

Let $b(\cdot, \cdot)$ be a bounded sesquilinear form defined in $V \times V$ and satisfying Gårding's inequality

$$
\operatorname{Re} b(v, v) \geq c_{0}\|v\|^{2}-c_{1}|v|^{2}, \quad c_{0}>0, \quad c_{1} \geq 0 .
$$

Let $A_{0}$ be the operator associated with the sesquilinear form $-b(\cdot, \cdot)$ :

$$
\left(A_{0} v_{1}, v_{2}\right)=-b\left(v_{1}, v_{2}\right), \quad v_{1}, v_{2} \in V .
$$

$A_{0}$ is a bounded linear operator from $V$ to $V^{*}$, and its realization in $H$ which is the restriction of $A_{0}$ to

$$
D\left(A_{0}\right)=\left\{v \in V ; A_{0} v \in H\right\}
$$

is also denoted by $A_{0}$. Then $A_{0}$ generates an analytic semigroup in both of $H$ and $V^{*}($ see $[7])$.

The operators $A_{1}$ and $A_{2}$ are bounded linear operators from $V$ to $V^{*}$ such that their restrictions to $D\left(A_{0}\right)$ are bounded linear operators from $D\left(A_{0}\right)$ equipped with the graph norm of $A_{0}$ to $H$. The function $a(\cdot)$ is assumed to be real valued and belongs to $L^{2}(-h, 0)$.

First, we consider some basic results on the following linear functional differential initial value problem:

$$
\left\{\begin{array}{l}
\frac{d}{d t} u(t)=A_{0} u(t)+A_{1} u(t-h)+\int_{-h}^{0} a(s) A_{2} u(t+s) d s+f(t), \\
u(0)=g^{0}, \quad u(s)=g^{1}(s) \quad-h \leq s \leq 0 .
\end{array}\right.
$$

By assumption there exists a positive constant $M_{0}$ such that

$$
|v| \leq M_{0}\|v\| \text {. }
$$

Then, for any $f \in H$ we have

$$
\|f\|_{*} \leq M_{0}|f|
$$

It follows from (2.1) that for $u \in V$

$$
\operatorname{Re}\left(\left(c_{1}-A_{0}\right) v, v\right) \geq c_{0}\|v\|^{2} .
$$

Hence there exists a constant $C_{0}$ such that

$$
\|v\| \leq C_{0}\|v\|_{D\left(A_{0}\right)}^{1 / 2}|v|^{1 / 2}
$$

for every $v \in D\left(A_{0}\right)$, where

$$
\|v\|_{D\left(A_{0}\right)}=\left(\left|A_{0} v\right|^{2}+|v|^{2}\right)^{1 / 2}
$$

is the graph norm of $D\left(A_{0}\right)$. 
If $X$ is a Banach space and $1<p<\infty, L^{p}(0, T ; X)$ is the collection of all strongly measurable functions from $(0, T)$ into $X$ whose $p$-th powers of norms are integrable and $W^{m, p}(0, T ; X)$ is the set of all functions $f$ whose derivatives $D^{\alpha} f$ up to degree $m$ in the distribution sense belong to $L^{p}(0, T ; X)$.

By virtue of Theorem 3.3 of [2] we have the following result on the corresponding linear equation of (LE).

Proposition 2.1. Suppose that the assumptions stated above are satisfied. Then the following properties hold:

1) Let $X=\left(D\left(A_{0}\right), H\right)_{1 / 2,2}$ where $\left(D\left(A_{0}\right), H\right)_{1 / 2,2}$ is the real interpolation space between $D\left(A_{0}\right)$ and $H\left(\right.$ see $\left[8\right.$, Section 1.3.3]). For $\left(g^{0}, g^{1}\right) \in X \times L^{2}(-h, 0$; $\left.D\left(A_{0}\right)\right)$ and $f \in L^{2}(0, T ; H), T>0$, there exists a unique solution $u$ of $(L E)$ belonging to

$$
\mathcal{W}_{0}(T) \equiv L^{2}\left(-h, T ; D\left(A_{0}\right)\right) \cap W^{1,2}(0, T ; H) \subset C([0, T] ; X)
$$

and satisfying

$$
\|u\|_{\mathcal{W}_{0}(T)} \leq C_{1}\left(\left\|g^{0}\right\|_{X}+\left\|g^{1}\right\|_{L^{2}\left(-h, 0 ; D\left(A_{0}\right)\right)}+\|f\|_{L^{2}(0, T ; H)}\right),
$$

where $C_{1}$ is a constant depending on $T$.

2) Let $\left(g^{0}, g^{1}\right) \in H \times L^{2}(-h, 0 ; V)$ and $f \in L^{2}\left(0, T ; V^{*}\right), T>0$. Then there exists a unique solution $u$ of $(L E)$ in case $G(\cdot, u) \equiv 0$ belonging to

$$
\mathcal{W}_{1}(T) \equiv L^{2}(-h, T ; V) \cap W^{1,2}\left(0, T ; V^{*}\right) \subset C([0, T] ; H)
$$

and satisfying

$$
\|u\|_{\mathcal{W}_{1}(T)} \leq C_{1}\left(\left|g^{0}\right|+\left\|g^{1}\right\|_{L^{2}(-h, 0 ; V)}+\|f\|_{L^{2}\left(0, T ; V^{*}\right)}\right),
$$

where $C_{1}$ is a constant depending on $T$.

Given $u \in L^{2}(0, T ; V)$ we extend it to the space $L^{2}(-h, T ; V)$ by setting $u(s)=g^{1}(s)$ for $s \in(-h, 0)$.

We assume the following hypotheses on the nonlinear mappings $F, k$ in (SLE):

(A1) $F:[0, T] \times L^{2}(0, T ; V) \times H \rightarrow H$ is a nonlinear mapping such that for $\phi \in L^{2}(0, T ; V)$ and $x \in H, F(t, \phi, x)$ is strongly measurable on $[0, T]$ and there exist positive constants $L_{0}, L_{1}, L_{2}$ and $L_{3}$ such that

$$
\left|F\left(t, \phi_{1}, x_{1}\right)-F\left(t, \phi_{2}, x_{2}\right)\right| \leq L_{1}|| \phi_{1}-\phi_{2}||+L_{2}\left|x_{1}-x_{2}\right|, \quad t \in[0, T] .
$$

(A2) Let $\Delta_{T}=\{(s, t): 0 \leq s \leq t \leq T\}$. Then $k: \Delta_{T} \times L^{2}(0, T ; V) \rightarrow H$ is a nonlinear mapping such that for $x \in H, k(t, s, x)$ is strongly measurable on $\Delta_{T}$ and there exists positive constant $L_{3}$ such that

$$
\left|k\left(t, s, x_{1}\right)-k\left(t, s, x_{2}\right)\right| \leq L_{3}|| x_{1}-x_{2} \|, \quad(s, t) \in \Delta_{T} .
$$

Remark 2.2. The above operator $F$ is the semilinear case of the nonlinear part of quasilinear equations considered by Yong and Pan [9]. 
For $u \in L^{2}(-h, T ; V), T>0$ we set

$$
G(t, u)=F\left(t, u(t-h), \int_{0}^{t} k(t, s, u(s-h)) d s\right) .
$$

Lemma 2.3. Let $u \in L^{2}(-h, T ; V) T>0$. Then $G(\cdot, u) \in L^{2}(0, T ; H)$ and

$$
\|G(\cdot, u)\|_{L^{2}(0, T ; H)} \leq L_{0} \sqrt{T}+\left(L_{1}+L_{2} L_{3} T / \sqrt{2}\right)\|u\|_{L^{2}(-h, T-h ; V)} .
$$

Moreover if $u_{1}, u_{2} \in L^{2}(-h, T ; V)$, then

(2.8) $\left\|G\left(\cdot, u_{1}\right)-G\left(\cdot, u_{2}\right)\right\|_{L^{2}(0, T ; H)} \leq\left(L_{1}+L_{2} L_{3} T / \sqrt{2}\right)\left\|u_{1}-u_{2}\right\|_{L^{2}(-h, T-h ; V)}$.

Proof. For $u \in L^{2}(-h, T ; V)$, since

$$
\begin{aligned}
\int_{0}^{T}\left|\int_{0}^{t} k(t, s, u(s-h)) d s\right|^{2} d t & \leq L_{3}^{2} \int_{0}^{T}\left(\int_{0}^{t}\|u(s-h)\| d s\right)^{2} d t \\
& \leq L_{3}^{2} \int_{0}^{T} t \int_{0}^{t}\|u(s-h)\|^{2} d s d t \\
& \leq L_{3}^{2} \frac{T^{2}}{2} \int_{0}^{T}\|u(s-h)\|^{2} d s
\end{aligned}
$$

from (A1) and (A2), it is easily seen that

$$
\begin{aligned}
& \|G(\cdot, u)\|_{L^{2}(0, T ; H)}=\left\{\int_{0}^{T}\left|F\left(t, u(t-h), \int_{0}^{t} k(t, s, u(s-h)) d s\right)\right|^{2} d t\right\}^{1 / 2} \\
& =\left\{\int_{0}^{T}\left|F\left(t, u(t-h), \int_{0}^{t} k(t, s, u(s-h)) d s\right)-F(t, 0,0)+F(t, 0,0)\right|^{2} d t\right\}^{1 / 2} \\
& \quad \leq\left\{\int_{0}^{T}\left|F\left(t, u(t-h), \int_{0}^{t} k(t, s, u(s-h)) d s\right)-F(t, 0,0)\right|^{2} d t\right\}^{1 / 2}+L_{0} \sqrt{T} \\
& \leq L_{0} \sqrt{T}+L_{1}\|u\|_{L^{2}(-h, T-h ; V)}+L_{2}\left\{\int_{0}^{T}\left|\int_{0}^{t} k(t, s, u(s-h)) d s\right|^{2} d t\right\}^{1 / 2} .
\end{aligned}
$$

The proof of (2.8) is similar.

Now we are ready to give the following result on the local solvability of (SLE).

Theorem 2.4. Suppose that the assumptions (A1), (A2) and (A3) are satisfied. Then for any $\left(g^{0}, g^{1}\right) \in H \times L^{2}(-h, 0 ; V)$ and $f \in L^{2}\left(0, T ; V^{*}\right), T>0$, there exists a time $T_{0}>0$ such that the functional differential equation (SLE) admits a unique solution $u$ in $\mathcal{W}_{1}\left(T_{0}\right) \equiv L^{2}\left(-h, T_{0} ; V\right) \cap W^{1,2}\left(0, T_{0} ; V^{*}\right)$.

Proof. Let us fix $T_{0}>0$ so that

$$
M:=C_{0} C_{1}\left(L_{1}+L_{2} L_{3} T_{0} / \sqrt{2}\right)\left(T_{0} / \sqrt{2}\right)^{1 / 2}<1,
$$


where $C_{0}$ and $C_{1}$ are constants in (2.4) and (2.5) respectively. Let $w$ be the solution of

$$
\begin{aligned}
\frac{d}{d t} w(t)= & A_{0} w(t)+A_{1} w(t-h) \\
& +\int_{-h}^{0} a(s) A_{2} w(t+s) d s+G(t, v)+f(t), \\
w(0)= & g^{0}, \quad w(s)=g^{1}(s), \quad s \in[-h, 0) .
\end{aligned}
$$

We are going to show that $v \mapsto w$ is strictly contractive from $L^{2}\left(0, T_{0} ; V\right)$ to itself if the condition (2.9) is satisfied. Let $w_{1}, w_{2}$ be the solutions of (2.10), (2.11) with $v$ replaced by $v_{1}, v_{2} \in L^{2}\left(0, T_{0} ; V\right)$, respectively. From $(2.5)$ and (2.8) it follows that

$$
\begin{aligned}
\left\|w_{1}-w_{2}\right\|_{L^{2}\left(0, T_{0} ; D\left(A_{0}\right)\right) \cap W^{1,2}\left(0, T_{0} ; H\right)} & \leq C_{1}\left\|G\left(\cdot, v_{1}\right)-G\left(\cdot, v_{2}\right)\right\|_{L^{2}\left(0, T_{0} ; H\right)} \\
& \leq C_{1}\left(L_{1}+L_{2} L_{3} \frac{T_{0}}{\sqrt{2}}\right)\left\|v_{1}-v_{2}\right\|_{L^{2}\left(0, T_{0} ; V\right)},
\end{aligned}
$$

and hence in view of (2.4) we have

$$
\begin{aligned}
& \left\|w_{1}-w_{2}\right\|_{L^{2}\left(0, T_{0} ; V\right)} \\
\leq & C_{0}\left\|w_{1}-w_{2}\right\|_{L^{2}\left(0, T_{0} ; D\left(A_{0}\right)\right)}^{\frac{1}{2}}\left\|w_{1}-w_{2}\right\|_{L^{2}\left(0, T_{0} ; H\right)}^{\frac{1}{2}} \\
\leq & C_{0}\left\|w_{1}-w_{2}\right\|_{L^{2}\left(0, T_{0} ; D\left(A_{0}\right)\right)}^{\frac{1}{2}}\left(\frac{T_{0}}{\sqrt{2}}\right)^{\frac{1}{2}}\left\|w_{1}-w_{2}\right\|_{W^{1,2}\left(0, T_{0} ; H\right)}^{\frac{1}{2}} \\
\leq & C_{0}\left(\frac{T_{0}}{\sqrt{2}}\right)^{\frac{1}{2}}\left\|w_{1}-w_{2}\right\|_{L^{2}\left(0, T_{0} ; D\left(A_{0}\right)\right) \cap W^{1,2}\left(0, T_{0} ; H\right)} \\
\leq & C_{0} C_{1}\left(L_{1}+L_{2} L_{3} \frac{T_{0}}{\sqrt{2}}\right)\left(\frac{T_{0}}{\sqrt{2}}\right)^{1 / 2}\left\|v_{1}-v_{2}\right\|_{L^{2}\left(0, T_{0} ; V\right)} .
\end{aligned}
$$

Here we used the following inequality

$$
\begin{aligned}
\left\|w_{1}-w_{2}\right\|_{L^{2}\left(0, T_{0} ; H\right)} & =\left\{\int_{0}^{T_{0}}\left|w_{1}(t)-w_{2}(t)\right|^{2} d t\right\}^{\frac{1}{2}} \\
& =\left\{\int_{0}^{T_{0}}\left|\int_{0}^{t}\left(\dot{w}_{1}(\tau)-\dot{w}_{2}(\tau)\right) d \tau\right|^{2} d t\right\}^{\frac{1}{2}} \\
& \leq\left\{\int_{0}^{T_{0}} t \int_{0}^{t}\left|\dot{w}_{1}(\tau)-\dot{w}_{2}(\tau)\right|^{2} d \tau d t\right\}^{\frac{1}{2}} \\
& \leq \frac{T_{0}}{\sqrt{2}}\left\|w_{1}-w_{2}\right\|_{W^{1,2}\left(0, T_{0} ; H\right)} .
\end{aligned}
$$

So by virtue of (2.9) the contraction mapping principle gives that equation (SLE) has a unique solution in $\left[-h, T_{0}\right]$. 


\section{Global existence and behavior of solution}

In this section we give norm estimate of the solution of (SLE) and which is helpful to establish the global existence of solutions with the aid of norm estimations.

Theorem 3.1. Suppose that the assumptions (A1), (A2) and (A3) are satisfied. Then for any $\left(g^{0}, g^{1}\right) \in H \times L^{2}(-h, 0 ; V)$ and $f \in L^{2}\left(0, T ; V^{*}\right), T>0$, the solution $u$ of $(S L E)$ exists and is unique in $\mathcal{W}_{1}(T) \equiv L^{2}(-h, T ; V) \cap W^{1,2}\left(0, T ; V^{*}\right)$, and there exists a constant $C_{2}$ depending on $T$ such that

$$
\|u\|_{\mathcal{W}_{1}(T)} \leq C_{2}\left(1+\left|g^{0}\right|+\left\|g^{1}\right\|_{L^{2}(-h, 0 ; V)}+\|f\|_{L^{2}\left(0, T ; V^{*}\right)}\right) .
$$

Proof. Let $u(\cdot)$ be the solution of (SLE) in the interval $\left[-h, T_{0}\right]$ where $T_{0}$ is a constant in $(2.9)$ and $w(\cdot)$ be the solution of the following equation

$$
\begin{aligned}
\frac{d}{d t} w(t) & =A_{0} w(t)+A_{1} w(t-h)+\int_{-h}^{0} a(s) A_{2} w(t+s) d s+f(t), \\
w(0) & =g^{0}, \quad w(s)=g^{1}(s), \quad-h \leq s<0 .
\end{aligned}
$$

Then in view of (2.5), (2.7)

$$
\begin{aligned}
& \|u-w\|_{L^{2}\left(0, T_{0} ; D\left(A_{0}\right)\right) \cap W^{1,2}\left(0, T_{0} ; H\right)} \\
\leq & C_{1}\|G(\cdot, u)\|_{L^{2}\left(0, T_{0} ; H\right)} \\
\leq & C_{1}\left\{L_{0} \sqrt{T}_{0}+\left(L_{1}+L_{2} L_{3} T_{0} / \sqrt{2}\right)\left(\|u\|_{L^{2}\left(0, T_{0} ; V\right)}+\left\|g^{1}\right\|_{L^{2}(-h, 0 ; V)}\right)\right\} . \\
\leq & C_{1}\left\{L_{0} \sqrt{T}_{0}+\left(L_{1}+L_{2} L_{3} T_{0} / \sqrt{2}\right)\left(\|u-w\|_{L^{2}\left(0, T_{0} ; V\right)}+\|w\|_{L^{2}\left(0, T_{0} ; V\right)}\right.\right. \\
& \left.\left.\quad+\left\|g^{1}\right\|_{L^{2}(-h, 0 ; V)}\right)\right\} .
\end{aligned}
$$

Thus, arguing as in the proof of (2.12)

$$
\begin{aligned}
& \|u-w\|_{L^{2}\left(0, T_{0} ; V\right)} \\
\leq & C_{0}\left(\frac{T_{0}}{\sqrt{2}}\right)^{\frac{1}{2}}\|u-w\|_{L^{2}\left(0, T_{0} ; D\left(A_{0}\right)\right) \cap W^{1,2}\left(0, T_{0} ; H\right)} \\
\leq & C_{0}\left(\frac{T_{0}}{\sqrt{2}}\right)^{\frac{1}{2}} C_{1}\left\{L_{0} \sqrt{T}_{0}+\left(L_{1}+L_{2} L_{3} T_{0} / \sqrt{2}\right)\left(\|u-w\|_{L^{2}\left(0, T_{0} ; V\right)}\right.\right. \\
& \left.\left.+\|w\|_{L^{2}\left(0, T_{0} ; V\right)}+\left\|g^{1}\right\|_{L^{2}(-h, 0 ; V)}\right)\right\} .
\end{aligned}
$$

For brevity, set

$$
M:=C_{0} C_{1}\left(L_{1}+L_{2} L_{3} T_{0} / \sqrt{2}\right)\left(T_{0} / \sqrt{2}\right)^{1 / 2}
$$

in the sense of (2.9). Therefore, we have

$$
\begin{aligned}
& \|u-w\|_{L^{2}\left(0, T_{0} ; V\right)} \\
\leq & \frac{C_{0} C_{1} L_{0} \sqrt{T_{0}}\left(T_{0} / \sqrt{2}\right)^{1 / 2}+M\left(\|w\|_{L^{2}\left(0, T_{0} ; V\right)}+\left\|g^{1}\right\|_{L^{2}(-h, 0 ; V)}\right)}{1-M}
\end{aligned}
$$


and hence, with the aid of 2) of Proposition 2.1

$$
\begin{aligned}
& \|u\|_{L^{2}\left(0, T_{0} ; V\right)} \\
\leq & \frac{C_{0} C_{1} L_{0} \sqrt{T_{0}}\left(T_{0} / \sqrt{2}\right)^{1 / 2}}{1-M}+\frac{\|w\|_{L^{2}\left(0, T_{0} ; V\right)}+\left\|g^{1}\right\|_{L^{2}(-h, 0 ; V)}}{1-M} \\
\leq & \frac{C_{0} C_{1} L_{0} \sqrt{T_{0}}\left(T_{0} / \sqrt{2}\right)^{1 / 2}}{1-M} \\
& +\frac{1}{1-M}\left\{C_{1}\left(\left|g^{0}\right|+\left\|g^{1}\right\|_{L^{2}(-h, 0 ; V)}+\|f\|_{L^{2}\left(0, T_{0} ; V^{*}\right)}\right)+\left\|g^{1}\right\|_{L^{2}(-h, 0 ; V)}\right\} .
\end{aligned}
$$

On the other hand using (2.6), (2.3), (2.7) we get

$$
\begin{aligned}
& \|u\|_{\mathcal{W}_{1}\left(T_{0}\right)} \\
\leq & C\left(\left|g^{0}\right|+\left\|g^{1}\right\|_{L^{2}(-h, 0 ; V)}+\|G(\cdot, u)+f\|_{L^{2}\left(0, T_{0} ; V^{*}\right)}\right) \\
\leq & C\left(\left|g^{0}\right|+\left\|g^{1}\right\|_{L^{2}(-h, 0 ; V)}+M_{0}\|G(\cdot, u)\|_{L^{2}\left(0, T_{0} ; H\right)}+\|f\|_{L^{2}\left(0, T_{0} ; V^{*}\right)}\right) \\
\leq & C\left[\left|g^{0}\right|+\left\|g^{1}\right\|_{L^{2}(-h, 0 ; V)}+\|f\|_{L^{2}\left(0, T_{0} ; V^{*}\right)}\right. \\
& \left.+M_{0}\left\{L_{0} \sqrt{T}_{0}+\left(L_{1}+L_{2} L_{3} T_{0} / \sqrt{2}\right)\left(\|u\|_{L^{2}\left(0, T_{0} ; V\right)}+\left\|g^{1}\right\|_{L^{2}(-h, 0 ; V)}\right)\right\}\right]
\end{aligned}
$$

for some constant $C$. Combining (3.2), and (3.3) we obtain

$$
\|u\|_{\mathcal{W}_{1}\left(T_{0}\right)} \leq C\left(1+\left|g^{0}\right|+\left\|g^{1}\right\|_{L^{2}(-h, 0 ; V)}+\|f\|_{L^{2}\left(0, T_{0} ; V^{*}\right)}\right)
$$

for some constant $C_{2}$. Since the condition (2.9) is independent of the initial values, the solution of (SLE) can be extended to the interval $\left[-h, n T_{0}\right]$ for every natural number $n$. An estimate analogous to (3.4) holds for the solution in $\left[-h, n T_{0}\right]$, and hence for the initial value $\left(u\left(n T_{0}\right), u_{n T_{0}}\right)$ in the interval $\left[n T_{0},(n+\right.$ 1) $\left.T_{0}\right]$.

Theorem 3.2. Suppose that the assumptions (A1), (A2) and (A3) are satisfied. If $\left(g^{0}, g^{1}\right) \in X \times L^{2}\left(-h, 0 ; D\left(A_{0}\right)\right)$ and $f \in L^{2}(0, T ; H)$, then $u \in \mathcal{W}_{0}(T) \equiv$ $L^{2}\left(-h, T ; D\left(A_{0}\right)\right) \cap W^{1,2}(0, T ; H)$, and the mapping $\left(g^{0}, g^{1}, f\right) \mapsto u \in \mathcal{W}_{0}(T)$ is continuous.

Proof. It is easy to show that if $\left(g^{0}, g^{1}\right) \in X \times L^{2}\left(-h, 0 ; D\left(A_{0}\right)\right)$ and $f \in$ $L^{2}(0, T ; H)$, then from Proposition 2.1 it follows that $u$ belongs to $\mathcal{W}_{0}(T)$. Let $\left(g_{i}^{0}, g_{i}^{1}, f_{i}\right) \in X \times L^{2}\left(-h, 0 ; D\left(A_{0}\right)\right) \times L^{2}(0, T ; H)$, and $u_{i}$ be the solution of (SLE) with $\left(g_{i}^{0}, g_{i}^{1}, f_{i}\right)$ in place of $\left(g^{0}, g^{1}, f\right)$ for $i=1,2$. Then in view of Proposition 2.1 and Lemma 2.3 we have

$$
\begin{aligned}
& \left\|u_{1}-u_{2}\right\|_{\left.\mathcal{W}_{0}(T)\right)} \\
\leq & C_{1}\left\{\left\|g_{1}^{0}-g_{2}^{0}\right\|_{X}\right. \\
& +\left\|g_{1}^{1}-g_{2}^{1}\right\|_{L^{2}\left(-h, 0: D\left(A_{0}\right)\right)}+\left\|G\left(\cdot, u_{1}\right)-G\left(\cdot, u_{2}\right)\right\|_{L^{2}(0, T ; H)} \\
& \left.+\left\|f_{1}-f_{2}\right\|_{L^{2}(0, T ; H)}\right\}
\end{aligned}
$$




$$
\begin{aligned}
\leq & C_{1}\left\{\left\|g_{1}^{0}-g_{2}^{0}\right\|_{X}+\left\|g_{1}^{1}-g_{2}^{1}\right\|_{L^{2}\left(-h, 0: D\left(A_{0}\right)\right)}+\left\|f_{1}-f_{2}\right\|_{L^{2}(0, T ; H)}\right. \\
& \left.+\left(L_{1}+L_{2} L_{3} T / \sqrt{2}\right)\left(\left\|u_{1}-u_{2}\right\|_{L^{2}(0, T: V)}+\left\|g_{1}^{1}-g_{2}^{1}\right\|_{L^{2}(-h, 0 ; V)}\right)\right\} .
\end{aligned}
$$

Since

we get

$$
u_{1}(t)-u_{2}(t)=g_{1}^{0}-g_{2}^{0}+\int_{0}^{t}\left(\dot{u}_{1}(s)-\dot{u}_{2}(s)\right) d s
$$

$$
|| u_{1}-\left.u_{2}\right|_{L^{2}(0, T ; H)} \leq \sqrt{T}\left|g_{0}^{1}-g_{2}^{0}\right|+\frac{T}{\sqrt{2}}|| u_{1}-u_{2} \|_{W^{1,2}(0, T ; H)} .
$$

Hence, arguing as in (2.12) we get

$$
\begin{aligned}
\leq & C_{0}\left\|u_{1}-u_{2}\right\|_{L^{2}\left(0, T ; D\left(A_{0}\right)\right)}^{1 / 2}|| u_{1}-u_{2} \|_{L^{2}(0, T ; H)}^{1 / 2} \\
\leq & C_{0}|| u_{1}-u_{2} \|_{L^{2}\left(0, T ; D\left(A_{0}\right)\right)}^{1 / 2} \\
& \times\left\{T^{1 / 4}\left|g_{1}^{0}-g_{2}^{0}\right|^{1 / 2}+\left(\frac{T}{\sqrt{2}}\right)^{1 / 2}|| u_{1}-u_{2} \|_{W^{1,2}(0, T ; H)}^{1 / 2}\right\} \\
\leq & C_{0} T^{1 / 4}\left|g_{1}^{0}-g_{2}^{0}\right|^{1 / 2}|| u_{1}-\left.u_{2}\right|_{L^{2}\left(0, T ; D\left(A_{0}\right)\right)} ^{1 / 2}+C_{0}\left(\frac{T}{\sqrt{2}}\right)^{1 / 2}\left\|u_{1}-u_{2}\right\|_{\mathcal{W}_{0}(T)} \\
\leq & 2^{-7 / 4} C_{0}\left|g_{1}^{0}-g_{2}^{0}\right|+2 C_{0}\left(\frac{T}{\sqrt{2}}\right)^{1 / 2}\left\|u_{1}-u_{2}\right\|_{\mathcal{W}_{0}(T)} .
\end{aligned}
$$

Combining (3.5) and (3.6) we obtain

$$
\begin{aligned}
& \left\|u_{1}-u_{2}\right\|_{\mathcal{W}_{0}(T)} \\
\leq & C_{1}\left\{\left\|g_{1}^{0}-g_{2}^{0}\right\|_{X}+\left\|g_{1}^{1}-g_{2}^{1}\right\|_{L^{2}\left(-h, 0: D\left(A_{0}\right)\right)}\right. \\
& \left.+\left\|f_{1}-f_{2}\right\|_{L^{2}(0, T ; H)}+\left(L_{1}+L_{2} L_{3} T / \sqrt{2}\right)\left\|g_{1}^{1}-g_{2}^{1}\right\|_{\left.L^{(-h, 0 ; V)}\right)}\right\} \\
& +2^{-7 / 4} C_{0} C_{1}\left(L_{1}+L_{2} L_{3} T / \sqrt{2}\right)\left|g_{1}^{0}-g_{2}^{0}\right|+2 C_{0} C_{1}\left(\frac{T}{\sqrt{2}}\right)^{1 / 2} \\
& \times\left(L_{1}+L_{2} L_{3} T / \sqrt{2}\right)\left\|u_{1}-u_{2}\right\|_{\mathcal{W}_{0}(T)} .
\end{aligned}
$$

Suppose that $\left(g_{n}^{0}, g_{n}^{1}, f_{n}\right) \rightarrow\left(g^{0}, g^{1}, f\right)$ in $X \times L^{2}\left(-h, 0 ; D\left(A_{0}\right)\right) \times L^{2}(0, T ; H)$, and let $u_{n}$ and $u$ be the solutions (SLE) with $\left(g_{n}^{0}, g_{n}^{1}, f_{n}\right)$ and $\left(g^{0}, g^{1}, f\right)$ respectively. Let $0<T_{1} \leq T$ be such that

$$
2 C_{0} C_{1}\left(T_{1} / \sqrt{2}\right)^{1 / 2}\left(L_{1}+L_{2} L_{3} T_{1} / \sqrt{2}\right)<1 .
$$

Then by virtue of (3.7) with $T$ replaced by $T_{1}$ we see that $u_{n} \rightarrow u$ in $\mathcal{W}_{0}\left(T_{1}\right)$. This implies that $\left(u_{n}\left(T_{1}\right),\left(u_{n}\right)_{T_{1}}\right) \mapsto\left(u\left(T_{1}\right), u_{T_{1}}\right)$ in $X \times L^{2}\left(-h, 0 ; D\left(A_{0}\right)\right)$. Hence the same argument shows that $u_{n} \rightarrow u$ in

$$
L^{2}\left(T_{1}, \min \left\{2 T_{1}, T\right\} ; D\left(A_{0}\right)\right) \cap W^{1,2}\left(T_{1}, \min \left\{2 T_{1}, T\right\} ; H\right) .
$$

Repeating this process we conclude that $u_{n} \rightarrow u$ in $\mathcal{W}_{0}(T)$. 
Theorem 3.3. For $f \in L^{2}(0, T ; H)$ let $u_{f}$ be the solution of equation (SLE). Let us assume that the embedding $D\left(A_{0}\right) \subset V$ is compact. Then the mapping $f \mapsto u_{f}$ is compact from $L^{2}(0, T ; H)$ to $L^{2}(0, T ; V)$.

Proof. If $f \in L^{2}(0, T ; H)$, then in view of Theorem 3.1

$$
\left\|u_{f}\right\|_{\mathcal{W}_{1}(T)} \leq C_{2}\left(1+\left|g^{0}\right|+\left\|g^{1}\right\|_{L^{2}(-h, 0 ; V)}+M_{0}\|f\|_{L^{2}(0, T ; H)}\right) .
$$

Since $u_{f} \in L^{2}(0, T ; V), G\left(\cdot, u_{f}\right) \in L^{2}(0, T ; H)$. Consequently $u_{f} \in L^{2}(0, T$; $D\left(A_{0}\right) \cap W^{1,2}\left(0, T^{*} ; V\right)$ and with aid of Proposition 2.1, Lemma 2.3, and (3.8),

$$
\begin{aligned}
& \left\|u_{f}\right\|_{L^{2}\left(0, T ; D\left(A_{0}\right) \cap W^{1,2}(0, T ; V)\right.} \\
\leq & C_{1}\left(\left\|g^{0}\right\|_{X}+\left\|g^{1}\right\|_{L^{2}\left(-h, 0 ; D\left(A_{0}\right)\right)}+\left\|G\left(\cdot, u_{f}\right)+f\right\|_{L^{2}(0, T ; H)}\right) \\
\leq & C_{1}\left\{\left\|g^{0}\right\|_{X}+\left\|g^{1}\right\|_{L^{2}\left(-h, 0 ; D\left(A_{0}\right)\right)}+L_{0} \sqrt{T}\right. \\
& \left.+\left(L_{1}+L_{2} L_{3} T / \sqrt{2}\right)\|u\|_{L^{2}(-h, T-h ; V)}+\|f\|_{L^{2}(0, T ; H)}\right\} \\
\leq & C_{1}\left[\left\|g^{0}\right\|_{X}+\left\|g^{1}\right\|_{L^{2}\left(-h, 0 ; D\left(A_{0}\right)\right)}+L_{0} \sqrt{T}\right. \\
& +\left(L_{1}+L_{2} L_{3} T / \sqrt{2}\right)\left\{\left\|g^{1}\right\|_{L^{2}(-h, 0 ; V)}+C_{2}\left(1+M_{0}\|f\|_{L^{2}(0, T ; H)}\right)\right\} \\
& \left.+\|f\|_{L^{2}(0, T ; H)}\right] .
\end{aligned}
$$

Hence if $f$ is bounded in $L^{2}(0, T ; H)$, then so is $u_{f}$ in $L^{2}\left(0, T ; D\left(A_{0}\right)\right) \cap$ $W^{1,2}(0, T ; H)$. Since $D\left(A_{0}\right)$ is compactly embedded in $V$ by assumption, the embedding

$$
L^{2}\left(0, T ; D\left(A_{0}\right) \cap W^{1,2}(0, T ; V) \subset L^{2}(0, T ; V)\right.
$$

is compact in view of Theorem 2 of J. P. Aubin [1].

\section{References}

[1] J. P. Aubin, Un thèoréme de compacité, C. R. Acad. Sci. Paris 256 (1963), 5042-5044.

[2] G. Di Blasio, K. Kunisch, and E. Sinestrari, $L^{2}$-regularity for parabolic partial integrodifferential equations with delay in the highest-order derivatives, J. Math. Anal. Appl. 102 (1984), no. 1, 38-57.

[3] W. E. Fitzgibbon, Semilinear integro-differential equations in Banach space, Nonlinear Anal. 4 (1980), no. 4, 745-760.

[4] M. L. Heard, An abstract semilinear hyperbolic Volterra integro-differential equation, J. Math. Anal. Appl. 80 (1981), no. 1, 175-202.

[5] J. M. Jeong, S. Nakagiri, and H. Tanabe, Structural operators anf semigroups associated with functional-differential equations in Hilbert space, Osaka J. Math. 30 (1993), no. 3, 365-395.

[6] J. M. Jeong, Retarded functional-differential equations with $L^{1}$-valued controller, Funkcial. Ekvac. 36 (1993), no. 1, 71-93.

[7] H. Tanabe, Fundamental solutions of differential equation with time delay in Banach space, Funkcial. Ekvac. 35 (1992), no. 1, 149-177.

[8] H. Triebel, Interpolation Theory, Function Spaces, Differential Operators, NorthHolland, 1978.

[9] J. Yong and L. Pan, Quasi-linear parabolic partial differential equations with delays in the highest order spatial derivatives, J. Austral. Math. Soc. Ser. A 54 (1993), no. 2, 174-203. 
Jin-Mun JEONG

Department of Applied Mathematics

Pukyong National University

Busan 608-737, Korea

E-mail address: jmjeong@pknu.ac.kr

Su Jin CheON

Department of Applied Mathematics

Pukyong National University

Busan 608-737, KoreA

E-mail address: hjwh12@hanmail.net 